\title{
El vidrio en la civilización egipcia. Descripción de piezas de vidrio del tipo incrustaciones de una colección de la Universidad de Sevilla
}

\author{
M. SECO ALVAREZ(*), A. RUIZ CONDE, M.A. VILlEGAS(**), P.J. SANCHEZ SOTO \\ Instituto de Ciencia de Materiales de Sevilla, Centro Mixto Consejo Superior de Investigaciones Científicas (CSIC)-Universidad de Sevilla, \\ c/ Américo Vespucio s/n, 41012 Isla de la Cartuja, Sevilla \\ $(*)$ Centre d'Etudes Alexandrines, Alejandría (Egipto) \\ (**) Centro Nacional de Investigaciones Metalúrgicas (CENIM), CSIC. 28040-Madrid
}

\begin{abstract}
En el presente trabajo, se destaca la importancia que la manufactura y el arte del vidrio tuvo para la civilización egipcia. Se describe a grandes rasgos su evolución histórica y los principales métodos de fabricación. A continuación, se resalta la existencia de una serie de materiales vítreos, denominados genéricamente incrustaciones, que en la época egipcia tuvieron diversos fines, todos ellos de elevado nivel artístico, como son pequeños amuletos, piezas de joyería y otras aplicaciones, entre ellos las máscaras y los cartonajes. Dichos materiales podrían ser precedentes del mosaico vidriado. Como ejemplo de interés de este tipo de manufactura, se han seleccionado una serie de incrustaciones en vidrio procedentes de una colección de Arte Egipcio de la Universidad de Sevilla con el objetivo de darla a conocer. Se describen dichas piezas y se lleva a cabo una discusión crítica sobre las características de las mismas. Los resultados obtenidos en la descripción de estas piezas se relacionan con información disponible en una serie de fuentes bibliográficas sobre incrustaciones, por ejemplo las de vidrio monocromático del Museo Grecorromano de Alejandría, Museo Británico y Museo Egipcio de El Cairo.
\end{abstract}

Palabras Clave: vidrios, incrustaciones, arqueometría, Egipto, Patrimonio Cultural

The glass in the Egyptian civilization. Description of glass elements considered as glass inlays of an Egyptian Collection of the University of Sevilla

In this work, the authors emphasize the importance that the manufacture and art of glass making represented for the Egyptian civilization. It is described the historical evolution of glass in Egypt and the main fabrication methods. Next, it is pointed out the existence of a particular class of materials, named as glass inlays. For the Egyptian period, these materials had several applications, all these of high art level, such as small amulets, jewellry, pieces and components of masks and cardboard articles. These materials could be the precursors of glass mosaics. As examples of the relevant interest in this kind of manufacture, it has been selected a particular Egyptian Collection of the University of Sevilla containing some pieces of glass inlays. The objective of this work is to provide valuable information to a broad community concerning these materials. Selected pieces are described and a critical discussion is performed considering the characteristics of each one. The results obtained in the description of these glass materials have been related with the information provided from selected bibliography concerning important glass collections, in particular of glass inlays. Between them, the authors included the monochromatic glass collection of The Greco-Roman Museum of Alexandria (Egypt), British Museum (United Kingdom) and Egyptian Museum of Cairo (Egypt).

Key Words: glass, glass inlays, archaeometry, Egypt, Cultural Heritage

\section{INTRODUCCION: EL VIDRIO Y SU FABRICACION EN EGIPTO}

Los vidrios y vidriados manufacturados en el antiguo Egipto están básicamente constituidos del mismo material, siendo su aplicación la principal diferencia entre ambos grupos (1-3). El vidriado más antiguo se fecha en el 12.000 A.C. y parece que procede de Asia, encontrándose restos de objetos de vidrio en Mesopotamia y Bagdad (2). La técnica de los vidriados se conocía ya por la civilización egipcia alrededor del 9.000 A.C. (1-6). Esto se ha demostrado por la existencia de figurillas de arena recubiertas de capas vítreas de color verde. Un amuleto azul oscuro realizado en pasta de vidrio se ha fechado en el 7.000 A.C. (6). Los vidrios arqueológicos más antiguos se remontan a vidriados y vidrios egipcios datados alrededor del 2.500 A.C. Se supone que estos productos de artesanía procedían de Siria $(2,3)$. La primera pieza de vidrio egipcio que se ha situado históricamente con certeza data del año 1.500 A.C. y se trata de una perla de vidrio azul turquesa (6). La manufactura del vidrio en Egipto alcanzó su máximo esplendor entre 1587 y 1327 A.C. y a partir del último milenio, la vidriería egipcia comparte con la zona de Siria la primacía en la producción. Los vidrios se utilizaron independientemente y los vidriados se realizaban sobre otro material, generalmente un soporte cerámico. 
Los joyeros egipcios, desde los inicios de esta civilización, realizaron muy variados intentos de reproducir el color de las piedras preciosas, como el lapislázuli y la turquesa (4-6). Estos intentos son la base del desarrollo de los vidriados verdosos y azulados, pastas vítreas e incluso podría ser del propio vidrio como aplicación ornamental. Por esta razón, los vidrios más antiguos están coloreados y muy raramente se encuentran vidrios incoloros y transparentes. Aparte del vidrio de color blanco lechoso o negro, se producían vidrios en colores como el azul intenso, azul celeste, violeta, verde, amarillo, ocre, anaranjado e incluso rojo o púrpura (con manganeso). En las primeras dinastías se manufacturaron los vidrios de color blanco, negro y púrpura; el amarillo y el rojo se utilizaron más profusamente a partir de la dinastía XVIII, cuando las composiciones vidriadas polícromas alcanzaron su mayor desarrollo. Algunos materiales vidriados con forma rectangular, similares a los azulejos $(6 \times 4 \mathrm{~cm})$ y de color verde o azul, son conocidos desde la dinastía III, por ejemplo en la pirámide de Saqqara. Algunos materiales de mayor tamaño de este tipo parecen ser modelados a mano a partir de una pasta de grano grueso, dándoles el tamaño adecuado y secando previamente antes de añadir el vidriado y someterlos a la cocción. Desde una perspectiva histórica (2-6), el vidrio en sí no fue producido en cantidad en Egipto hasta el reinado del faraón Amenhotep III (Amenophis III) durante la XVIII dinastía (1552-1305 A.C.) en el Imperio Nuevo. Un impulso nuevo condujo a la manufactura de vasos de vidrio durante el reinado de Tutmosis III (14901436 A.C.), probablemente debido al contacto con fabricantes asiáticos de vidrio durante las campañas militares de los egipcios en la zona de Siria.

De forma general, la fabricación del vidrio en Egipto partía de materias primas útiles en la preparación de una pasta más o menos vítrea. Del proceso de fabricación se han realizado documentados estudios (1-6). Una composición apta para la obtención de vidrio estaba constituida básicamente por mezclas de arena de sílice $\left(\mathrm{SiO}_{2}\right)$, el mineral natrón (carbonato sódico) y piedra caliza molida (carbonato de calcio, $\mathrm{CaCO}_{3}$ ). Esta mezcla se calentaba en bruto a temperaturas de $1000-1100^{\circ} \mathrm{C}$. También se ha mencionado la utilización de cenizas de plantas una vez calcinadas y que aportan álcalis a la mezcla $(2,6)$. Previamente, o durante el proceso, se procedía a realizar un molde con la forma deseada, se cubría con un trozo de tela y se fijaba a un vástago o varilla de metal. A continuación, se introducía el molde en la masa de componentes minerales que ya estaría fundida y se daban vueltas con rapidez para que el vidriado se extendiera del modo más uniforme posible. Si el vidrio estaba todavía blando por efecto de la temperatura, podía decorarse aplicando encima una o varias varillas delgadas de vidrio coloreado, previamente preparadas, que rodeaban el objeto en espiral (por ejemplo, el caso típico de una vasija). Estas varillas delgadas, en estado pastoso, se utilizaban para decoración. Por medio de herramientas metálicas, como agujas o peines, se podían trazar diversos motivos decorativos, tales como ondas, arcos, dibujos, cenefas, etc. Una vez calentado todo el conjunto otra vez, se alisaba la superficie haciendo girar el objeto sobre un bloque compacto de piedra. Finalmente, una vez enfriado el objeto, se sacaba el molde interior.

El vidrio también se podía introducir una vez fundido en la cavidad de un molde. Una vez igualada la superficie y terminada la cocción, se extraía de dicho molde. Si se trataba de elaborar objetos más complejos, se utilizaba la técnica de la cera perdida, ampliamente conocida y empleada en la obtención de piezas metálicas y en la manufactura de joyas $(1,4,5)$. Es de destacar que la adaptación de las técnicas de metalurgia a la fabricación de piezas de vidrio consistió en llenar el molde con una composición de vidrio previamente molido que se fundía con el calor del horno y de este modo rellenaba el espacio vacío que dejaba la cera por efecto del aumento de temperatura.

Como se ha comentado anteriormente, la elaboración del vidrio posee conexiones importantes con la de los metales (4-6). Por tanto, no es de extrañar la adaptación de métodos metalúrgicos a la fabricación del vidrio, tal como el prensado en caliente. Las propias escorias procedentes de la obtención y refino de metales están constituidas por silicatos complejos e impuros, además de residuos minerales, la mayor parte de las veces formando masa esponjosas o compactas con aspecto vítreo. En este sentido, la aparición del vidrio podría haber tenido su origen como consecuencia del proceso de obtención y procesado de los metales, que a su vez requiere del concurso de materiales cerámicos (por la época y la tecnología, silicoaluminosos) con el propósito de realizar crisoles, toberas, etc. para su manufactura. Ciertas composiciones vidriadas consisten en una parte interna o núcleo de granos de arena de cuarzo muy pura, cuarzo en masa o cristal de roca y cuarzo molido que adquiere cierta consistencia o coherencia al ser enlazados los granos mayores con una disolución del mineral natrón y ser sometido el conjunto a un periodo de tratamiento térmico. De esta forma tan simple se fabricaban pequeños objetos de adorno, pendientes, collares, anillos y otros, como son algunos objetos funerarios (amuletos y estatuillas).

Aparte de las técnicas anteriormente mencionadas, la civilización egipcia desarrolló el arte del tallado en vidrio, de modo similar al utilizado en la manufactura de piezas para joyería (4-6). Se torneaban y tallaban en frío bloques de vidrio. La elaboración del vidrio en Egipto era todo un arte, pero suponía un trabajo agotador y muy perjudicial para la salud de trabajadores y artesanos por los riesgos del trabajo con hornos a temperaturas elevadas $\left(700-1000{ }^{\circ} \mathrm{C}\right)$, humos del horno, vapores procedentes del vidrio fundido y otros que podían originar quemaduras y graves daños.

\section{PIEZAS DE VIDRIO EGIPCIO: INCRUSTACIONES Y AMULETOS}

Las piezas más frecuentes de vidrio egipcio son: a) recipientes pequeños de vidrio hueco, moldeados por la técnica del núcleo de arena (por ejemplo, vasos, jarros, ánforas, ungüentarios, etc.); b) vasos y cuencos obtenidos por prensado en caliente dentro de un molde; c) recipientes manufacturados por la técnica del tallado y grabado, semejante al procedimiento usado con las piedras duras; d) vasos, cuencos y recipientes obtenidos por la técnica "mille fiori" (mil flores) o vidrios mosaico, uniendo distintas piezas de vidrio y pasta vítrea colorea$\mathrm{da}$, previamente preparadas con varillas de vidrio de distintos colores que se reunían, se ablandaban por efecto del calor y se cortaban en discos o placas delgadas; posteriormente se soldaban al fuego para formar vasos, cuencos $u$ otros recipientes formando un conjunto o composición artística de gran efecto de policromía $(2,6)$.

Bajo la denominación de incrustaciones, se pueden considerar aquéllas piezas de vidrio que decoran diversos sarcófagos de madera, cartonajes ptolemaicos y romanos, o bien amuletos. La aparición en Egipto de las primeras incrustaciones conoci- 
das en vidrio datan de la XVIII dinastía (1552-1305 A.C.), en el periodo denominado Imperio Nuevo. Existen muchos ejemplos descritos con anterioridad y pueden mencionarse algunos de ellos. Uno de los más conocidos son las incrustaciones que decoran el sarcófago de madera de Petosiris y su hermano (7) y los utilizados para decorar los cartonajes desde época ptolemaica hasta romana (9). Estas pequeñas piezas de vidrio se podrían considerar también como amuletos aunque no lleven un enganche. Según Schlick-Nolte (10), los amuletos en vidrio, desde la época ptolemaica a la romana, no solían llevarlo pues se encontraban envueltos con la momia. Sin embargo, hay que tener en cuenta que los amuletos en vidrio, a pesar de que existieron y son conocidos desde el reinado del faraón Amenhotep III, no fueron realmente muy numerosos. A partir de la XXX dinastía (378-341 A.C.) su producción creció, aunque los fabricados en fayenza siguieron siendo los más abundantes (1-3).

La gran innovación que se registra fue el desarrollo de amuletos-relieves realizados en vidrios transparentes. Para su manufactura, primero se realizaban con un molde en el que se vertía una pequeña cantidad de vidrio fundido a partir de mezclas de materias primas como son natrón y arena silícea, además de materias colorantes, empleando crisoles de arcilla refractaria. Cuando la primera capa se había enfriado, se vertía otra de distinto color en el molde, por lo que resultaban muy decorativos. A veces se lograba un efecto de cristal opaco que se hace traslúcido con la luz (11). Por tanto, para determinar si son incrustaciones o amuletos habría que conocer su procedencia exacta, lo cual a veces resulta muy difícil.

La civilización egipcia alcanzó un elevado nivel artístico con los trabajos de incrustaciones (12). Ya en la época predinástica (finales del V milenio-3065 A.C.) se utilizaba esta técnica para hacer los ojos de las estatuas. Otras veces, las incrustaciones realizadas en vidrio o pasta de vidrio incrustada, se aplicaban a muebles o incluso a vasos de libaciones con un minucioso trabajo y con un gran efecto de policromía (12). Sobre todo, la realización de incrustaciones en vidrio y pasta vítrea tuvo un gran desarrollo dentro del mundo de las joyas, como ya se ha mencionado $(4,5,13)$. Dependiendo del caso, se podían fabricar en piedra, fayenza, vidrio, oro o marfil $(4,5,14)$. A partir del S. IV, se experimenta una gran riqueza de colorido y un gran avance de la técnica, que no es comparable al de ningún otro lugar a finales de la época clásica. Esto conduce a admitir la idea de que son los griegos del Egipto ptolemaico los que introducirán el uso del mosaico vidriado. Al final de la época helenística aparecen las piezas de mayor perfección y finura $(1-3,15)$.

\section{ELEMENTOS OBJETO DE LA DESCRIPCION}

Los elementos que se presentan en este trabajo como parte ilustrativa y decriptiva pertenecen a una colección mayor de piezas de época egipcia, procedente de Mr. Foudree (Profesor de facultad encargado de lengua inglesa), adquirida por el Departamento de Arqueología de la Universidad de Sevilla entre los años 1968-70. Se trata de una serie de piezas pequeñas, pero no por ello carecen de valor científico. Probablemente sean producto de exploraciones superficiales realizadas en excavaciones o adquiridas en el mercado de antigüedades, que en los años de la presencia inglesa en Egipto fue muy activo. De esta colección se ha seleccionado un conjunto de piezas de vidrios egipcios considerados incrustaciones (Fig. 1). En una primera

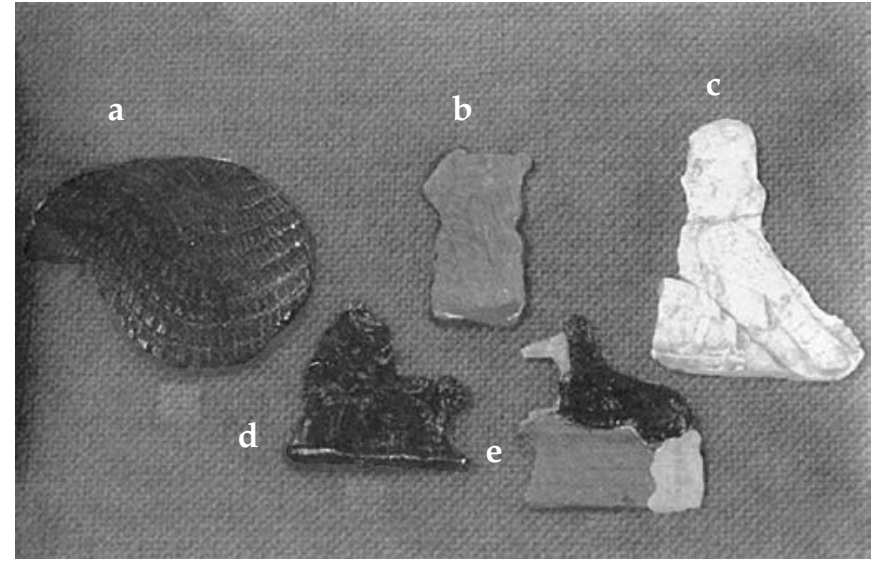

Figura 1.- Conjunto de las piezas de incrustaciones en vidrio de la colección egipcia: a) Peluca (vidrio azul); b) figurilla del dios Anubis como momificador (vidrio azul); c) Anubis como acompañante (vidrio azul claro); d) Anubis sobre su arqueta (azul claro, azul oscuro y amarillo); y e) Pájaro-Ba (vidrio verde muy claro)

fase del trabajo, se pretende dar a conocer una colección desconocida hasta ahora y que viene a enriquecer el Patrimonio de la Universidad de Sevilla. En otra fase posterior, se aplicarán métodos instrumentales no destructivos para un análisis más profundo de los materiales constituyentes.

Los datos obtenidos en la descripción de las piezas de esta colección se relacionan con la información disponible en otras colecciones más importantes que existen de incrustaciones, por ejemplo las de vidrio monocromático del Museo Grecorromano de Alejandría, Museo Británico y Museo Egipcio de El Cairo.

\section{DESCRIPCION DE LOS ELEMENTOS ESTUDIADOS Y DISCUSION DE SUS CARACTERISTICAS}

Se han seleccionado una serie de pequeñas piezas, como son Peluca (vidrio azul), figurillas del dios Anubis (vidrio azul, azul claro, azul oscuro y amarillo) y Pájaro-Ba (vidrio verde muy claro), a modo de ejemplos ilustrativos de incrustaciones en vidrio (Fig. 1). En lo que sigue, se describirán dichas piezas de incrustaciones en vidrio y se realizará una discusión sobre las características de las mismas que servirán de base para estudios posteriores de las piezas empleando métodos instrumentales.

\subsection{Peluca}

Se trata de una pieza completa. Sus medidas son: altura $35 \mathrm{~mm}$; ancho $43 \mathrm{~mm}$; grosor $7 \mathrm{~mm}$. Es de vidrio azul y de la época egipcia de Amarna (1364-1346 A.C.).

Es un trabajo de incrustación muy fino con la representación de una peluca rizada (Fig. 1) de color azul intenso, lo que es característico de las pelucas de la época de Amarna, de modo que esta incrustación en vidrio se puede fechar en ese periodo. Incrustaciones en vidrio de este tipo son muy numerosas (10-16). Existen modelos en el Tesoro de Tutankhamon (faraón de la XVIII dinastía que reinó de 1346 a 1337 A.C.). El peinado con rizos se encuentra en muchas de estas piezas, tal 
como en el magnífico trabajo de la silla dorada del trono de dicho faraón. Por ejemplo, la peluca procedente de Tebas y que se conserva en el Museo Británico (16) y clasificada como pieza EA 2280. Otra pieza similar de incrustación en vidrio es una peluca de mujer de la XVIII dinastía también con el mismo peinado de rizos, descrita en una colección privada (17).

Es muy difícil determinar si esta peluca (Fig. 1) perteneció a la figura de un hombre o a una mujer. Si se considera que está completa, pertenecería a un hombre y en la parte delantera parece que hay los restos de haber llevado un ureo, es decir, una pequeña figura de la diosa serpiente, elemento muy habitual en el arte egipcio $(1,4,5)$. Si se considera incompleta, podría ser la parte superior de una peluca femenina. En la famosa silla dorada con incrustaciones en vidrio del tesoro de Tutankhamon, ya mencionada, puede comprobarse la existencia de una pieza muy parecida a la descrita con el característico color azul intenso (18). Tanto el faraón como su esposa Anksenamón aparecen representados en este trabajo minucioso de incrustación con una peluca muy similar a la descrita.

Ha de indicarse que en la fabricación de vidrios coloreados del mismo tipo que el mencionado, se añadía una materia colorante que generalmente era un compuesto de cobre que formaba vidrios azules e incluso verdes. Algunos resultados de análisis químico en este tipo de vidrios indican la presencia del elemento cobalto, sólo o con cobre $(2,4)$. Los compuestos conteniendo cobalto eran materias primas importadas de Egipto. Tanto el cobalto como el cobre son elementos que si están presentes generan coloraciones azules en vidrios y vidriados (por ejemplo, recuérdese el color azul cobalto en típicos azulejos vidriados).

\subsection{Figura del dios Anubis como momificador}

Se trata de una pieza incompleta, falta la muñeca y la mano derecha. Sus medidas son: altura $26 \mathrm{~mm}$; ancho $26 \mathrm{~mm}$; grosor $4 \mathrm{~mm}$. Es de vidrio azul, de época egipcia desde XXX dinastía (378-341 A.C.) hasta época ptolemaica/romana.

La pieza de vidrio representa al dios Anubis con cuerpo humano y cabeza de chacal (Fig. 1). Se encuentra de pié, de lado y con una pierna adelantada. Con la mano derecha toca al momificado y la izquierda la tiene levantada. Delante de él está la momia echada sobre un lecho con forma de león, con la cabeza, las patas y la cola elevada. Anubis, dios egipcio del cementerio y del embalsamamiento, lo tenemos representado como momificador (1-5). Este tuvo que embalsamar el cuerpo del dios Osiris y también el del muerto transformado en Osiris. Así, existen multitud de escenas en las que se manifiesta a dicho dios ejerciendo su función (Fig. 2). Esta típica imagen se reprodujo en amuletos de la época ptolemaica y éstos, para que el muerto se beneficiara de las ventajas de la momificación (19), se colocaban en las piernas y pecho del difunto.

En el Museo Egipcio de El Cairo (18) existen dos ejemplos muy parecidos al estudiado. Uno procede de la zona de Sakkara, en el que se ve claramente cómo Anubis sostiene el corazón del difunto sobre la palma de la mano izquierda (20). Por tanto, aunque este detalle falte en la incrustación en vidrio estudiada, puede suponerse que quizás lo llevaría. El otro ejemplo está realizado en vidrio opaco de color blanco (21). Otra pieza muy similar a la estudiada es un amuleto en vidrio procedente de la zona de Dendara; está realizado en vidrio azul y con pintura negra, fechándolo Petrie (22) en época ptolemaica.

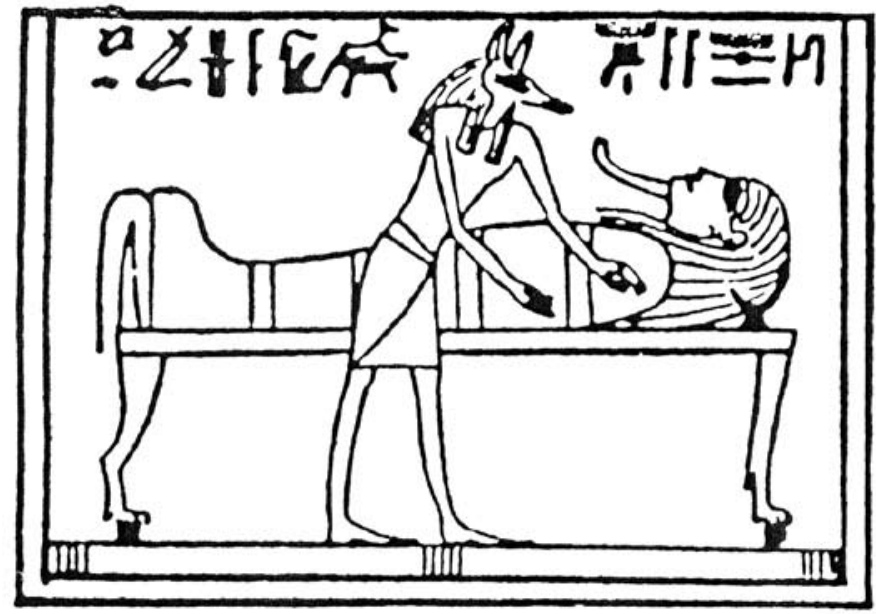

Figura 2.- Representación iconográfica del dios Anubis con cabeza de chacal como momificador.

\subsection{Anubis como acompañante}

Se trata de una pieza incompleta, falta una pequeña lasca de la parte superior de la cabeza y de la ofrenda. Altura $29 \mathrm{~mm}$; ancho $16 \mathrm{~mm}$; grosor $4 \mathrm{~mm}$. Vidrio azul. Epoca egipcia: época tardía (400-300 A.C.).

Se ve al dios Anubis con cabeza de chacal y cuerpo humano, de pié con una pierna adelantada y mirando hacia la derecha (Fig. 1). El brazo izquierdo lo tiene estirado hacia abajo y el derecho vuelto hacia arriba. Sobre la mano elevada lleva una bandeja con un objeto y parece estar haciendo una ofrenda. No se observa con claridad qué es lo que lleva sobre dicha bandeja, pero podría tratarse del corazón del difunto, como corresponde a muchas iconografías (1-5). Existen algunos ejemplos bastante similares al estudiado, procedentes de Sakkara y que se conservan en el Museo Egipcio de El Cairo (22,23). Uno de ellos es el que más se asemeja a esta pieza de incrustación en vidrio y se observa que representa al dios Anubis exactamente en la misma posición. En este caso, sí se distingue que sobre la mano derecha lleva el corazón del difunto. Estas piezas están catalogadas como amuletos y no como incrustaciones en vidrio, dado que se encontraron envueltas junto a la momia (24).

\subsection{Anubis sobre su arqueta}

En este caso, se considera una pieza completa pero partida en dos trozos. Altura $29 \mathrm{~mm}$; ancho $26 \mathrm{~mm}$; grosor $4 \mathrm{~mm}$. Realizado en vidrio azul claro, azul oscuro y vidrio amarillo. Epoca egipcia: época ptolemaica (332-30 A.C.).

Se trata de una pieza de incrustación en vidrio con la representación del dios Anubis como un chacal sentado sobre su arqueta (Fig. 1). Se encuentra mirando hacia la derecha. Una línea horizontal que abarca desde la parte alta de la cola hasta las patas delanteras del animal corta al vidrio en dos fragmentos. Un paralelo muy similar se encuentra con otra pieza que se conserva en el Museo Egipcio de El Cairo (25). En ese caso concreto, el chacal mira hacia la izquierda y lo que varía es 
el arca sobre el que está recostado. Otro ejemplo, también de época ptolemaica y procedente de Akhmin, se conserva en el Museo Británico (10). Es un relieve de color verde esmeralda con la representación del chacal echado.

Como ya se mencionó previamente, para determinar con una exactitud relativa si se trata de una incrustación o de un amuleto habría que saber el lugar exacto de su procedencia. Se han descrito casos en los que figuras del dios Anubis se representan sobre su arqueta y se consideran amuletos $(26,27)$. Sin embargo, en otras ocasiones son incrustaciones en vidrio (28).

\subsection{Figura de pájaro-ba}

Esta incrustación en vidrio una pieza incompleta: el vidrio está partido por dos lugares. El primero es una línea horizontal que corta al pájaro-ba a la altura del cuello; el segundo es una línea transversal que corta la parte baja de las patas del animal. Altura $39 \mathrm{~mm}$; ancho $16 \mathrm{~mm}$; grosor $4 \mathrm{~mm}$. Realizado en vidrio verde muy claro, casi blanco. Epoca egipcia: época ptolemaica (332-30 A.C.).

Se trata de una incrustación en vidrio con la representación de un $b a$ con el cuerpo en forma de ave, la cabeza de humano y coronado por el disco solar (Fig. 1). El trabajo realizado se aprecia que ha sido muy fino.

Reisner (29) menciona y describe algunos modelos parecidos que se conservan en el Museo Egipcio de El Cairo. Se pueden encontrar piezas rematadas por el disco solar o bien otras alternativas. Una de ellas es utilizar la doble corona en vez del disco solar, como se observa en algunos casos (piezas CG 13410 y 13412). O simplemente no se colocaba nada sobre la cabeza del $b a$, como por ejemplo en la pieza CG 13417. Esto está relacionado con los diferentes tipos de $b a$ que existían, de modo que el disco solar hacía referencia al ba del dios Ra, la doble corona al del faraón y si no aparecía nada podía ser el $b a$ de cualquier difunto. Nótese que para los egipcios el cuerpo era el componente físico de cada persona y se completaba con el ba y el ka. Así, el ba se consideraba como el espíritu o personalidad. Este, cuando la persona moría, tenía libertad para moverse tanto en el mundo de los muertos como en el de los vivos. Desde el Imperio Nuevo se representaba con cuerpo de pájaro y cabeza humana $(1,2)$, tal como se muestra en la Fig. 3.

Si este tipo de pieza se considera amuleto, al encontrarse junto a la momia, aparece a la altura del pecho, lo que debía simbolizar la unión con el cuerpo (30). El ba debía viajar, desplazarse sobre la tumba o salir fuera de ella. De ahí la representación como un pájaro con cabeza humana, aunque su destino era finalmente unirse de nuevo al cuerpo. Cuando el dios egipcio Chnum modelaba en su torno de alfarero a cada persona, junto con el cuerpo físico le proporcionaba otros elementos igual de importantes para su supervivencia: el $k a$, el $b a$ (también llamado a veces bai), el aj, el nombre y la sombra. El aj era la unión del ba y el ka. Lo que hoy llamaríamos el alma, el cuerpo y el espíritu, era para los egipcios el $b a$, el $k a$ y el $a j$. La destrucción de uno de ellos significaba, sin remisión, la destrucción y aniquilación de toda la persona.

\section{CONSIDERACIONES FINALES Y SUMARIO}

Cada vez son mayores los esfuerzos por realizar investigaciones sobre materiales que forman parte o están relacionados

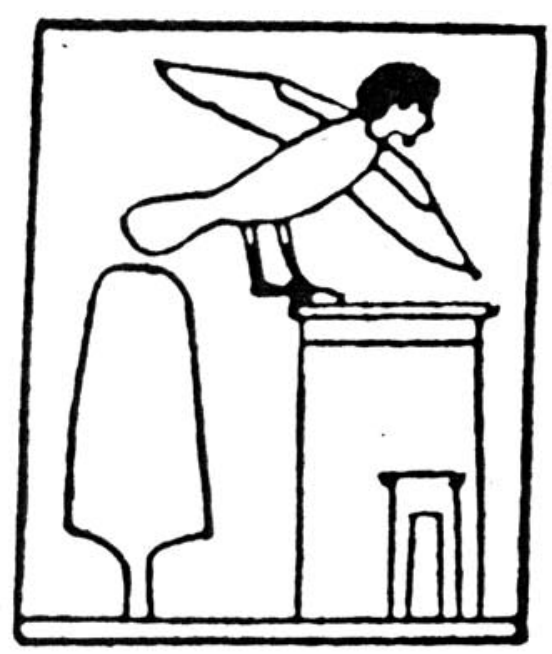

Figura 3.- Representación iconográfica egipcia del ba con cuerpo de pájaro y cabeza humana (el alma retornando a la tumba).

con el Patrimonio Histórico y Cultural, a pesar de sus limitaciones y muchas veces, su propia infravaloración. Al tratarse de vidrios, han sido escasos los estudios y con frecuencia se omiten en la relación de materiales de interés histórico y artístico o incluso no suelen tratarse en eventos científicos especializados, como son las reuniones sobre Arqueometría o Patrimonio Cultural. No obstante, en los últimos años se está corrigiendo esta tendencia y la comunidad científica, al menos en parte, se siente motivada hacia el estudio de los materiales que forman parte de los bienes culturales e históricos y de sus procesos físico-químicos de alteración. El patrimonio vidriero es muy variado y abundante $\mathrm{y}$, a pesar de ser un gran desconocido en Ciencia y Tecnología de Materiales, ocupa un lugar destacado en las ramas del conocimiento dedicadas a la Historia, el Arte y la Restauración. Es importante destacar que entre los diversos objetos de vidrio, las vidrieras y en especial las vidrieras medievales, se destacan como los bienes más conocidos y preciados $(31,32)$. La presente contribución está en la línea de comenzar a divulgar colecciones de Arte, en particular egipcio, en nuestro país y que son parte de su Patrimonio Cultural dentro de la Cerámica y el Vidrio. En este sentido, se menciona un reciente artículo publicado en este Boletín que muestra una panorámica de los vidrios castellanos de Cadalso de los Vidrios y de El Recuenco (33).

En primer lugar, en este artículo se ha querido poner de manifiesto la importancia que la manufactura y el arte del vidrio tuvo para la civilización egipcia como nuevo material a diferencia de los metales, materiales pétreos o cerámicos. En concreto, se ha querido resaltar la existencia de los materiales vítreos, algunos denominados genéricamente incrustaciones, y que en la época egipcia tuvieron diversos fines, todos ellos de un alto nivel artístico. Estos van desde la utilización como pequeños amuletos hasta su empleo en joyería y otras aplicaciones, como son las máscaras y los cartonajes. De todo su desarrollo posterior, una importante consecuencia es que se ha llegado a admitir la idea de que son los griegos del Egipto ptolemaico los que introducen el uso del mosaico vidriado. 
Como ejemplos de interés para su divulgación, se han seleccionado y descrito una serie de elementos considerados incrustaciones realizadas en vidrio, procedentes de una colección de pequeñas piezas de arte egipcio de la Universidad de Sevilla y que se pretende dar a conocer (34). Entre toda la colección, se pueden mencionar figuras votivas en bronce, recipientes de cerámica, fayenza y piedra, estatuillas funerarias (denominadas uschebtis) en alabastro, amuletos, sellos y otros objetos realizados en distintos materiales, así como objetos de piedra caliza con inscripciones (denominadas ostraca) y en madera. Una descripción de las piezas más interesantes de incrustaciones realizadas en vidrio se ha planteado a la hora de realizar este artículo a efectos divulgativos, llevándose a cabo una discusión crítica sobre sus características. Esta información servirá de base para estudios posteriores de estos materiales vítreos por medio de la utilización de métodos no destructivos de análisis de materiales. Para una mayor perspectiva, los resultados de la descripción realizada correspondiente a estas piezas de incrustaciones, se han relacionado con la información disponible en una serie de fuentes bibliográficas, lo que permite tener una idea global del conjunto en relación a los propios elementos vítreos considerados en el presente trabajo y los datos precedentes. Así, se han tenido en cuenta descripciones de importantes colecciones de incrustaciones, por ejemplo las de vidrio monocromático del Museo Grecorromano de Alejandría, Museo Británico y Museo Egipcio de El Cairo. No es de extrañar, pues, que piezas de incrustaciones en vidrio estén presentes en museos de nuestro país y todavía no hayan sido catalogadas como tales. Por último, es necesario indicar que para determinar si se trata de una incrustación o un amuleto realizados en vidrio de época egipcia habría que saber el lugar exacto de procedencia de la pieza, lo que no siempre es factible puesto que en algunos casos se trata de verdaderos amuletos y, en otros, incrustaciones realizadas en vidrio.

De todo ello se puede concluir que una colaboración entre equipos multidisciplinares de historiadores y de científicos del área de materiales, más concretamente de especialistas en cerámica y vidrio, es de gran interés en este tipo de estudios e investigaciones para conocer más en profundidad la cultura heredada de nuestros antepasados. La existencia de Redes Temáticas, como la constituida en el CSIC desde el mes de octubre de 2001, que se ocupa de aunar esfuerzos multidisciplinares en el estudio del Patrimonio Histórico y Cultural, es otro aspecto científico a considerar y que debería ser potenciado en un próximo futuro.

\section{AGRADECIMIENTOS}

Al Departamento de Prehistoria y Arqueología de la Universidad de Sevilla y al Instituto de Egiptología de la Universidad de Tübingen por su apoyo para la realización del estudio de las piezas catalogadas. Asimismo, se agradece la ayuda económica de la Junta de Andalucía al Grupo de Investigación TEP204 (Investigador Responsable: Dr. P.J. Sánchez Soto) dentro del III Plan Andaluz de Investigación y al Ministerio de Ciencia y Tecnología por la concesión del Proyecto MAT2002-02549, financiado en parte con FEDER. Trabajo realizado dentro de la Red Temática de Patrimonio Histórico y Cultural del CSIC.

\section{BIBLIOGRAFIA}

1. C. Aldred, Egyptian Art, London, 1980.

2. H. Tait (Editor), Five Thousand Years of Glass, London, 1991.

3. D. F. Grose, The Toledo Museum of Art. Early Ancient Glass, New York, 1989.

4. C. Aldred, Jewellery of the Pharaohs, London, 1978.

5. C. A. R. Andrews, Ancient Egyptian Jewellery, London, 1980.

6. J. M. ${ }^{a}$ Fernández Navarro, El Vidrio, Ed. CSIC y Centro Nacional del Vidrio, Madrid, 1991, págs. 4-14.

7. H. W. Müller, Äegyptische Kunstwerke, Kleinfunde und Glass in der Sammlung E. und M. Kofler-Truniger, p. 122 y fig. A186, Luzern, Berlin, 1964.

8. Por ejemplo, una cabeza de cartonaje en la Galería de Arte Walters de Baltimore (EE.UU.) y otra que se conserva en el Museo Egipcio de El Cairo; ver R.S. Bianchi, "Glass Inlays", Bulletin of the Egyptological Seminal, 5, p. 14, figs. 4 y 5, New York, 1983.

9. B. Schlick-Nolte, Liebieg-Museum Alter Plastik. Äegyptische Bildwerke, Vol. I, p. 278-279.

10. J. D. Cooney, Catalogue of Egyptian antiquities in the British Museum, Vol. IV, p. 1, Oxford, 1976.

11. W. Helck, "Einlegearbeiten", Lexikon der Ägyptologie I, p. 1207-1210, Wiesbaden, 1975.

12. Un ejemplo es un vaso de madera con incrustaciones en fayenza de la VI dinastía y que se conserva en el Museo de El Cairo; ver H. Kayser, Äegyptisches Kunsthandwerk, p. 124, fig. 111, Braunschweig, 1969.

13. Por ejemplo, en la tumba de Tuthankamon hay un gran número de pectorales con incrustaciones de vidrio opaco coloreado. Estos copian los pectorales del Imperio Medio, que se hacían con incrustaciones de piedras preciosas; ver A. Lucas, "Glass Figures", Annales du Service des Antiquitiés de l’Égypte, El Cairo, 39, p. 232, 1939; ver referencias (1) y (5).

14. J. Samson, Amarna, City of Akhenaten and Nefertiti, London, 1972

15. Un estudio de las incrustaciones en vidrio monocromo y de mosaicos del Museo Grecorromano de Alejandría está descrito en una monografía de Marie-Dominique Nenna, Etudes sur la verrerie clasique et Hellenistique en Mediterranée Orientale, París, 1992. Se trata del estudio de unas 400 piezas y son las procedentes de la necrópolis de Ras el-Tin donde se encuentran ejemplos de inspiración egipcia.

16. Fl. D. Friedman, Gifts of the Nile, Ancient Egyptian Fayence, p. 185 n128 y fig. en p. 83, London, 1998

17. Fl. D. Friedman, op. cit., p. 184 n126 y fig. en p. 83, London, 1998.

18. S. Donadoni, Le musée égyptian du Caire, p. 128-129, París, 1971.

19. Ver Friedman, op. cit., p. 244.

20. M. G. A. Reisner, Amulets, Vol. II, p. 130 y lám. 20, El Cairo, 1958.

21. M. G. A. Reisner, op. cit., lám. 31.

22. W. H. F. Petrie, Amulets, Illustrated by the Egyptian Collection in the University College London, p. 23 y lám. 7 (87c), Warminster, 1914.

23. Ver Reisner, op. cit., p. 28 y lám. 19.

24. H. W. Müller, Äegyptische Kunstwerke, Kleinfunde und Glass in der Sammlung E. und M. Kofler-Truniger, p. 123, Berlin, 1964. También en la ref. (2) se pueden encontrar numerosos ejemplos.

25. M. G. A. Reisner, op. cit., p. 129 y lám. 20.

26. N1 64096; ver Cooney, ref. (10), p. 10, fig. 94.

27. W. H. F. Petrie, op. cit., lám. 53, n119 y 23 ; los amuletos se encontraron depositados sobre la momia, dentro de dos sarcófagos procedentes de Dendera.

28. ver H. W. Müller, op. cit., láms. A 178 y A 179 a-b.

29. ver M. G. A. Reisner, op. cit., p. 113 y lám. 17; idem., p. 112-113 y lám. 17.

30. L. V. Zabkar, “Ba”, Lexikon der Ägyptologie I , p. 588-590, Wiesbaden, 1975.

31. J. M Mernández Navarro, "Procesos de alteración de las vidrieras medievales. Estudio y tratamientos de protección", Mater. Constr. 46 [242243] 3-25 (1996).

32. $\mathrm{M}^{\mathrm{a}} \mathrm{A}$. Villegas Broncano y M. García Heras, “El patrimonio vidriero. Técnicas de estudio", p. 24-37 en: Técnicas instrumentales y su aplicación al estudio del Patrimonio Histórico y Cultural. Curso Teórico-Práctico, Editores Científicos A. Ruiz Conde y P.J. Sánchez Soto, Gabinete de Formación del CSIC, Instituto de Ciencia de Materiales de Sevilla (CSIC-US), Imprenta COPYUR, Sevilla, 2002.

33. D. Belinchón Yagüe, "Vidrios castellanos de Cadalso de los Vidrios y de El Recuenco", Bol. Soc. Esp. Ceram. Vidr. 40 [5] 385-388 (2001).

34. M. Seco Alvarez, La Colección Egipcia de la Universidad Hispalense, Litografía Sáenz S.A., Sevilla, 2000.

Recibido: 07.02.02

Aceptado: 30.03 .03 